\title{
Article
}

\section{In-Target Proton-Boron Nuclear Fusion Using a PW-Class Laser}

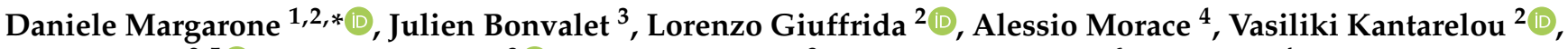 \\ Marco Tosca ${ }^{2,5}$ (), Didier Raffestin ${ }^{3}$ (), Philippe Nicolai ${ }^{3}$, Antonino Picciotto ${ }^{6}$, Yuki Abe ${ }^{4}$, Yasunobu Arikawa ${ }^{4}$, \\ Shinsuke Fujioka ${ }^{4}$, Yuji Fukuda ${ }^{7}$, Yasuhiro Kuramitsu ${ }^{8}$, Hideaki Habara ${ }^{8}$ and Dimitri Batani ${ }^{3,9}$
}

Citation: Margarone, D.; Bonvalet, J.; Giuffrida, L.; Morace, A.; Kantarelou, V.; Tosca, M.; Raffestin, D.; Nicolai, P.; Picciotto, A.; Abe, Y.; et al. In-Target Proton-Boron Nuclear Fusion Using a PW-Class Laser. Appl. Sci. 2022, 12, 1444. https://doi.org/10.3390/ app12031444

Academic Editor: Emilio Martines

Received: 21 December 2021

Accepted: 26 January 2022

Published: 28 January 2022

Publisher's Note: MDPI stays neutral with regard to jurisdictional claims in published maps and institutional affiliations.

Copyright: (c) 2022 by the authors. Licensee MDPI, Basel, Switzerland. This article is an open access article distributed under the terms and conditions of the Creative Commons Attribution (CC BY) license (https:// creativecommons.org/licenses/by/ $4.0 /)$.
1 Centre for Plasma Physics, School of Mathematics and Physics, Queen's University of Belfast, Belfast BT7 1NN, UK

2 ELI-Beamlines Center, Institute of Physics, Czech Academy of Sciences, Za Radnicí 835, 25241 Dolní Břežany, Czech Republic; Lorenzo.Giuffrida@eli-beams.eu (L.G.); Vasiliki.Kantarelou@eli-beams.eu (V.K.); Marco.Tosca@eli-beams.eu (M.T.)

3 CELIA (Centre Lasers Intenses et Applications), CNRS, CEA, Université de Bordeaux, UMR 5107, F-33405 Talence, France; julien.bonvalet@u-bordeaux.fr (J.B.); didier.raffestin@u-bordeaux.fr (D.R.); philippe.nicolai@u-bordeaux.fr (P.N.); dimitri.batani@u-bordeaux.fr (D.B.)

4 Institute of Laser Engineering, Osaka University, 2-6 Yamada-oka, Suita 565-0871, Japan; morace@ile.osaka-u.ac.jp (A.M.); abe.yuki@eei.eng.osaka-u.ac.jp (Y.A.); arikawa-y@ile.osaka-u.ac.jp (Y.A.); sfujioka@ile.osaka-u.ac.jp (S.F.)

5 Department of Macromolecular Physics, Faculty of Mathematics and Physics, Charles University, V Holešovičkách 2, 18000 Prague, Czech Republic

6 MNF-The Micro Nano Characterization and Fabrication Facility, Bruno Kessler Foundation, Via Sommarive 18, 38122 Trento, Italy; picciotto@fbk.eu

7 Kansai Photon Science Institute (KPSI), National Institutes for Quantum and Science and Technology (QST), 8-1-7 Umemidai, Kizugawa-shi 619-0215, Japan; fukuda.yuji@qst.go.jp

8 Graduate School of Engineering, Osaka University, 2-1 Yamada-oka, Suita 565-0871, Japan; kuramitsu@eei.eng.osaka-u.ac.jp (Y.K.); habara@eei.eng.osaka-u.ac.jp (H.H.)

9 HB11 Energy Holdings pty. Ltd., 11 Windora Avenue, Sydney, NSW 2096, Australia

* Correspondence: d.margarone@qub.ac.uk

\begin{abstract}
Nuclear reactions between protons and boron-11 nuclei ( $\mathrm{p}-\mathrm{B}$ fusion) that were used to yield energetic $\alpha$-particles were initiated in a plasma that was generated by the interaction between a PW-class laser operating at relativistic intensities $\left(\sim 3 \times 10^{19} \mathrm{~W} / \mathrm{cm}^{2}\right)$ and a $0.2-\mathrm{mm}$ thick boron nitride $(\mathrm{BN})$ target. A high $\mathrm{p}-\mathrm{B}$ fusion reaction rate and hence, a large $\alpha$-particle flux was generated and measured, thanks to a proton stream accelerated at the target's front surface. This was the first proof of principle experiment to demonstrate the efficient generation of $\alpha$-particles $\left(\sim 10^{10} / \mathrm{sr}\right)$ through p-B fusion reactions using a PW-class laser in the "in-target" geometry.
\end{abstract}

Keywords: proton-boron fusion; laser-plasma acceleration; $\alpha$-particle beam

\section{Introduction}

The conventional route of nuclear fusion for power generation is based on the reaction between deuterium and tritium nuclei, which yields one $\alpha$-particle and one neutron. Formidable technological challenges, however, stem from the production and handling of tritium, as well as from the radiation damage and radioactivity induced by the highenergy neutrons in the reactor materials. In this respect, the nuclear reaction between a proton and a boron-11 nucleus ( $\mathrm{p}-\mathrm{B}$ fusion) to yield three energetic $\alpha$-particles is very attractive, as it only involves abundant and stable isotopes in the reactants and there is no neutron in the reaction products. Previous studies have reported a main resonance of such nuclear reactions occurring for incoming proton beam energies at $675 \mathrm{keV} \mathrm{[1],}$ which shows a corresponding cross-section of about 1.2 barn. The $\alpha$-particles generated from $\mathrm{p}-\mathrm{B}$ fusion present a broad energy spectrum that peaks around $4 \mathrm{MeV}$ [1]; however, cutoff energies up to $10 \mathrm{MeV}$ have been demonstrated experimentally [2-6]. In the last 15 years, p-B fusion has been effectively induced by means of high-power lasers, which has 
reported an impressive progression in the reaction yield $[2,4,7,8]$, thus has become a point of interest for the energy sector where it is being considered as an alternative approach to conventional inertial confinement fusion schemes [9-11] and also potentially for medicine where intense $\alpha$-particle beams can be used for radioisotope production [12]. However, an extensive systematic investigation of laser-based $\mathrm{p}-\mathrm{B}$ fusion of the deep understanding of the underpinning physics is still missing [13]. An overview of the recent experimental progression in $\mathrm{p}-\mathrm{B}$ fusion in terms of $\alpha$-particle flux (or flux per input laser energy) is shown in Figure 1, both for the "in-target" [2-4,7,14] and "pitcher-catcher" geometries [5,6,8,15]. In this work, we show the first experimental results of efficient $\alpha$-particle production from p-B fusion using a PW-class laser in the "in-target" (i.e., direct irradiation) configuration. The results that were achieved during the same campaign in the "pitcher-catcher" geometry have been published elsewhere $[5,6]$.

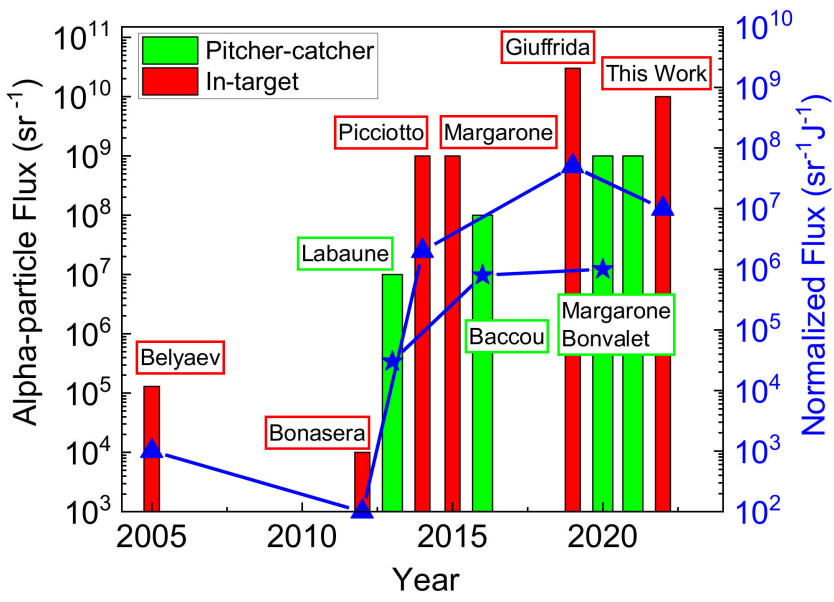

Figure 1. The experimental progress in $\mathrm{p}-\mathrm{B}$ fusion, measured in terms of $\alpha$-particle production in the "in-target" $[2-4,7,14]$ and "pitcher-catcher" $[5,6,8,15]$ geometries. The left-hand scale indicates the absolute $\alpha$-particle flux (particles/sr), while the right-hand scale is normalized to the laser energy delivered on target (particles/sr/J).

\section{Materials and Methods}

The relatively short-pulse (2.2 ps) and high-energy ( 1.4 kJ) PW-class laser system LFEX [16], which was operated at relativistic intensities $\left(\sim 3 \times 10^{19} \mathrm{~W} / \mathrm{cm}^{2}\right)$ at the Institute of Laser Engineering of the Osaka University (Japan), was focused onto the front surface (normal incidence) of a boron nitride (BN) target with a thickness of $0.2 \mathrm{~mm}$. The concentration of hydrogen in the sample was a few \%, which came from the chemical synthesis of the material during the manufacturing process.

As schematically shown in Figure 2, a Thomson parabola (TP) spectrometer was placed in the forward direction along the target normal to monitor the proton/ion plasma emission from the target's rear surface, which was based on an acceleration mechanism commonly known as "target normal sheath acceleration" (TNSA) [17]. Protons that were accelerated via TNSA at the target's rear side did not contribute to the generation of $\alpha$-particles from $\mathrm{p}-\mathrm{B}$ fusion; however, the determination of their cutoff energy was important to confirm that that particular laser shot was representative of an optimal laser-plasma coupling (a high laser intensity on the target's front surface allows the generation of electrons with high temperature, also known as "hot electrons", hence efficient TNSA at the target's rear side and protons with high cutoff energies). Plasma ions were deflected by parallel electric and magnetic fields based on their charge-to-mass ratio and were ultimately recorded on an imaging plate [18]. The presence of protons with cutoff energies of $\sim 25 \mathrm{MeV}$ confirmed that relativistic electrons were efficiently produced at the target's front side, thanks to the relatively high intensity and long pulse width of the incoming laser beam. The main $\alpha$-particle diagnostic was a CR39 nuclear track detector that was shielded with Al filters of different thicknesses $(10 \mu \mathrm{m}$ and $30 \mu \mathrm{m})$, which was aimed at the target's front side and 
placed at a distance of $144 \mathrm{~cm}$ from the target and at an angle of $\sim 80^{\circ}$ from the target normal. Such a large detection angle was deliberately chosen so that the CR39 sample would be out of the main blow-off plasma emission cone, thus excluding the presence of energetic heavy ions ( $\mathrm{B}$ and $\mathrm{N}$ ) emitted backwards and impinging on the detector. The calibration of the CR39 detectors with various Al filters is reported elsewhere, along with the etching procedure that was used in this work [6]. We note that tracks that were ascribable to the lowenergy blow-off plasma protons (very small pits in the CR39 sample) were unambiguously distinguishable from those ascribable to $\alpha$-particles from $\mathrm{p}-\mathrm{B}$ fusion events (larger pits) when the etching time was kept short enough $(\leq 1 \mathrm{~h})$, and that high-energy protons were not visible on the CR39 since they would have generated tracks with diameters below the resolution of the optical microscope that was used to map the sample after particle irradiation $(<1 \mu \mathrm{m})$.
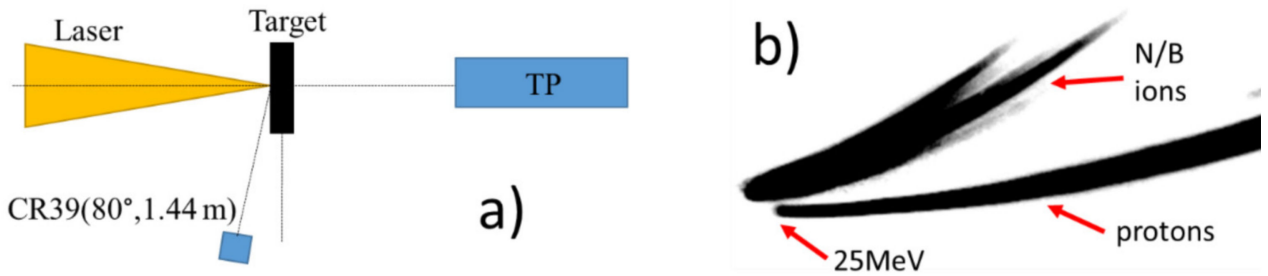

Figure 2. (a) The experimental setup; (b) the Thomson parabola (TP) snapshot showing the presence of protons and heavier ions being accelerated forwards from the target's rear surface (TNSA acceleration mechanism), i.e., not contributing to the generation of $\alpha$-particles via $\mathrm{p}-\mathrm{B}$ fusion.

A set of start-to-end numerical simulations was carried out with the aim of providing a qualitative interpretation of the experimental results. Additionally, 2D hydrodynamic simulations were performed using the CHIC code [19] with the aim of modeling the interaction of the relatively long ( $\sim \mathrm{ns})$ laser pedestal with the solid target. Then, 2D particle-in-cell (PIC) simulations were run using the SMILEI code [20] with the goal of modeling the acceleration of the protons at the target's front surface toward the target bulk (i.e., the protons moving forwards), thus highlighting the ongoing mechanism known as hole-boring radiation pressure acceleration (HB-RPA) [21,22]. The collision between the forward accelerated protons and the BN target bulk (assumed to be "cold" for simplicity) was modeled in 3D using the Monte Carlo FLUKA code $[23,24]$ with the aim of estimating the relative flux and energy distribution of the $\alpha$-particles that were generated by $p-B$ fusion events and propagated backward. Although the PIC simulation was performed in $2 \mathrm{D}$ due to computational constraints, this was a reasonable approximation since the HB-RPA that occurred at the target's front surface was weakly affected by the number of dimensions in the numerical simulation in terms of proton energies. In fact, the maximum proton energy was directly linked to the radiation pressure of the laser pulse at the center of the focal spot where the intensity was maximized (e.g., the proton energy calculated by 3D PIC simulations can be even larger than that in 2D PIC simulations) [25].

\section{Results}

The energy spectrum of the $\alpha$-particles that were emitted backward from the target's front surface is shown in Figure 3a. This energy distribution was recalculated from the signal recorded by the CR39 detectors that were covered by $10-\mu \mathrm{m}$ and $30-\mu \mathrm{m}$ Al filters (Figure 3b,c, respectively). The measured $\alpha$-particle flux in the energy range of 5-10 MeV and at the detection angle of $80^{\circ}$ (with respect to the target normal) was $1.2 \times 10^{10} / \mathrm{sr} \pm 17 \%$. This estimation was carried out by integrating the curve shown in Figure $3 a$ and using a reasonable extrapolation (the red dashed line in Figure $3 a$ ) of the spectrum between 7 and $8 \mathrm{MeV}$ (this was not measured due to the limitations of our calibration [6]). Particles with energy $<5 \mathrm{MeV}$ could not be detected since they were stopped in the $10-\mu \mathrm{m} \mathrm{Al}$ filter, and particles with energy potentially $>10 \mathrm{MeV}$ (diameter $<4 \mu \mathrm{m}$ ) were not counted since they were outside the CR39 calibration. 

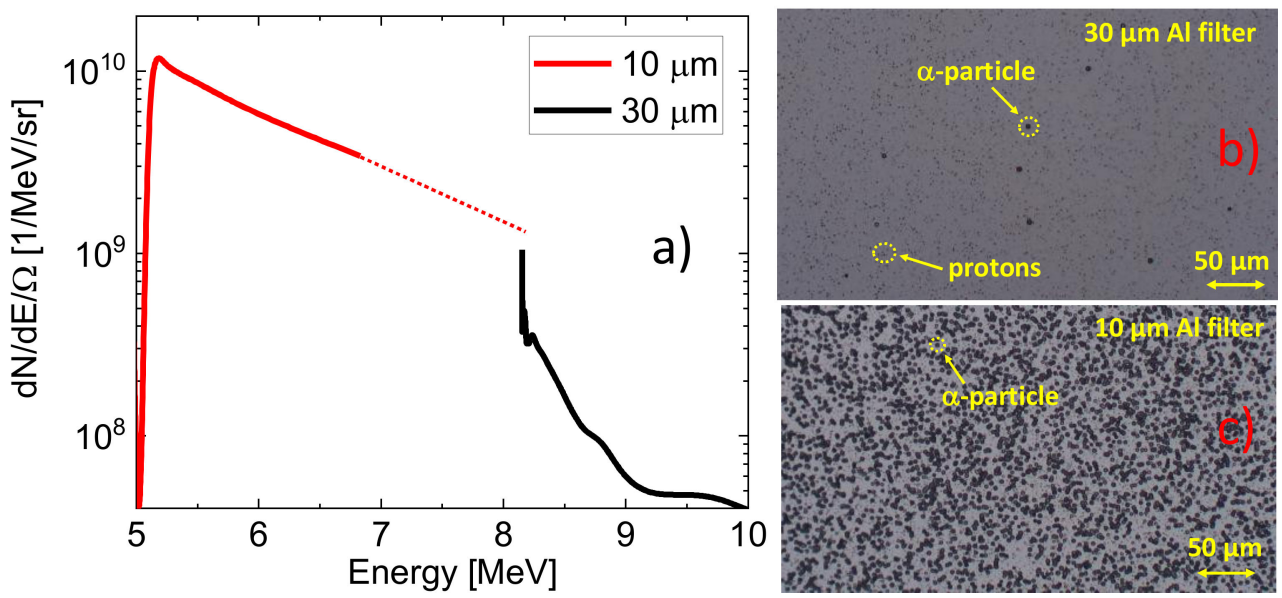

Figure 3. (a) The experimental spectrum of the $\alpha$-particles that were emitted in the backward direction from the target's front surface; (b) the corresponding CR39 raw image in the case of the 30- $\mu \mathrm{m} \mathrm{Al}$ filter and (c) the $10-\mu \mathrm{m} \mathrm{Al}$ filter.

The presence of a relatively large pre-plasma region that longitudinally extended for a length of $\sim 100 \mu \mathrm{m}$ in front of the target was estimated by the 2D hydrodynamic simulations. This information was implemented in the geometry that was used for the 2D PIC run, as shown in the proton density map of Figure 4a (the red dashed rectangle). The interface between the pre-plasma and the solid density region (around $175 \mu \mathrm{m}$ in Figure $4 \mathrm{a}$ ) along with the relatively long laser pulse feature ( $\sim 2 \mathrm{ps})$ allowed the onset of an effective HB-RPA process at the target's front surface [21,22], which ultimately led to the efficient acceleration of the protons that were propagating forward into the target bulk. This can be clearly seen in Figure $4 b$, which reports the proton phase space plot $\left(p_{x}>0\right)$. The energy distribution of the protons that were propagating forward, which was obtained from the 2D PIC simulation, is shown in Figure 4c (the black line). Protons with an energy of $0.5-19 \mathrm{MeV}$ were accelerated toward the $\mathrm{BN}$ target interior, thus generating $\mathrm{p}-\mathrm{B}$ fusion events inside the target. We noted that, according to the simulation outputs, the highest proton flux lay in the range of $0.5-1 \mathrm{MeV}$, which was an optimal condition for an efficient $\mathrm{p}-\mathrm{B}$ fusion process (the high cross-section of the nuclear reaction). Furthermore, the flux of protons with energies $>8 \mathrm{MeV}$ was relatively low, hence their contribution in terms of $\mathrm{p}-\mathrm{B}$ fusion yield was negligible (also due to the low cross-section of the nuclear reaction at such energies). Therefore, the low-energy part of the proton spectrum was responsible for the high-flux $\alpha$-particle streams that were propagating backward, as predicted by the 3D Monte Carlo simulation output shown in Figure $4 \mathrm{~d}$. The corresponding $\alpha$-particle energy distribution that was calculated by the Monte Carlo simulation for an angle corresponding to the position of the CR39 detector $\left(80^{\circ}\right)$ is reported in Figure $4 \mathrm{c}$ (the red line). The energy cutoff of the $\alpha$-particle stream that was calculated numerically was $\sim 14 \mathrm{MeV}$, but this could not be verified experimentally due to the limitations in the available $\alpha$-particle calibration.
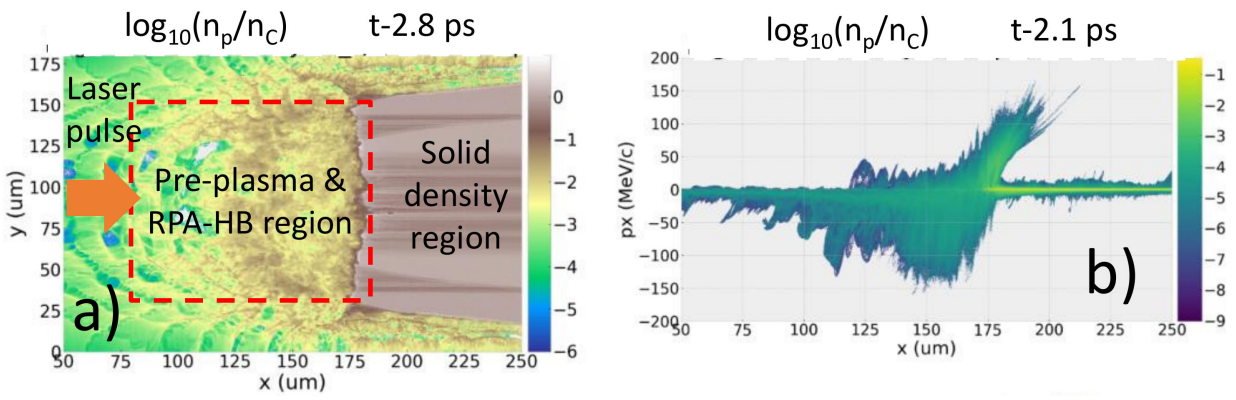

Figure 4. Cont. 

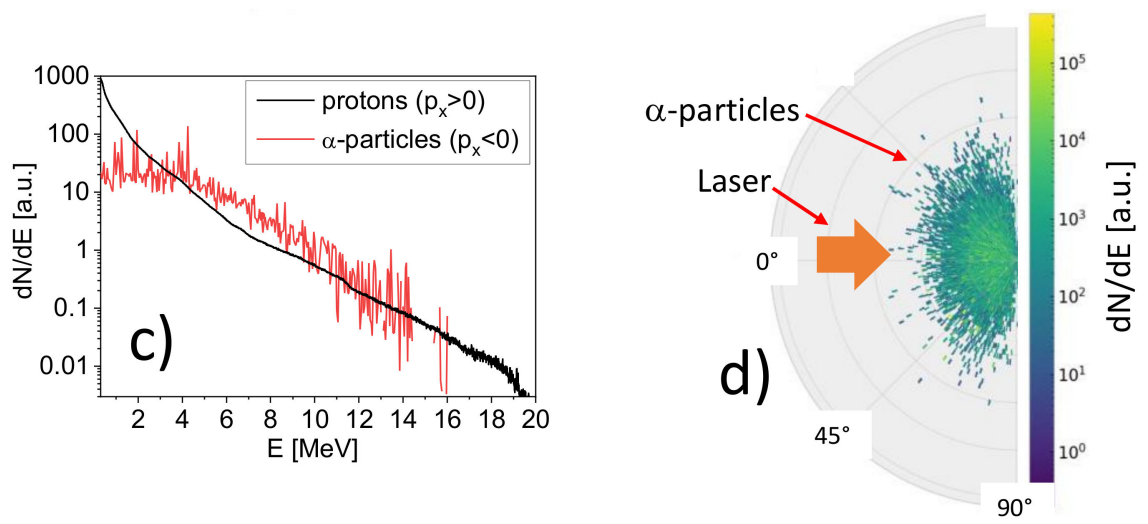

Figure 4. (a) The proton density map that was calculated by 2D PIC simulations at $t=2.8$ ps (i.e., 1.2 ps after the highest intensity peak entered the highest density part of the target); (b) the proton phase space plot at $t=2.1$ ps (the proton density is shown in units of plasma critical density); (c) the proton energy distribution $\left(p_{x}>0\right)$ and $\alpha$-particle energy distribution at the target's front side from PIC and Monte Carlo simulations, respectively; and (d) the $\alpha$-particle angular distribution from the same simulation run.

\section{Discussion and Conclusions}

The results presented in this work provide the first proof of principle experimental demonstration of efficient $\alpha$-particle generation from p-B fusion using a PW-class laser and the "in-target" geometry. The measured $\alpha$-particle flux was $\sim 10^{10} / \mathrm{sr}$, thus one order of magnitude higher than previous results that were obtained with the same laser parameters but in the "pitcher-catcher" geometry [5,6]. This achievement is in line with the experimental progress in $\mathrm{p}-\mathrm{B}$ fusion that has been reported in the last 15 years (see Figure 1) and confirms the advantage of triggering $\mathrm{p}-\mathrm{B}$ fusion reactions using a direct irradiation scheme, at least in terms of $\alpha$-particle flux [2-4]. A crude estimate of the total $\alpha$-particle generation could be provided under the assumption of quasi-isotropic emission, which was based on the fact that the kinetic energy of the accelerated protons was relatively low (unlike the pitcher-catcher geometry that was reported in our previous $\mathrm{p}-\mathrm{B}$ fusion experiment at LFEX [6]), hence there was no substantial momentum transfer from the protons to the $\alpha$-particles. Therefore, under such a rough assumption, the total number of $\alpha$-particles (including those particles absorbed inside the thick BN target) was $\sim 1.4 \times 10^{11}$. However, despite the high $\alpha$-particle flux that was experimentally measured, we noted that the overall conversion efficiency of the process (laser to $\alpha$-particle energy) was still low $(\sim 0.005 \%)$. It is worth noting that the $\alpha$-particle flux that was measured experimentally was a clear underestimation of the number of $\alpha$-particles that were emitted backward due to the limited energy range $(5-10 \mathrm{MeV})$ that was detectable by the diagnostics that were used. In fact, the numerically predicted $\alpha$-particle energy range was much broader $(1-14 \mathrm{MeV})$. Thus, considering the diagnostic limitations, we could expect a produced $\alpha$-particle flux and conversion efficiency in line with the previous results that were reported in [4] with a kJ (TW-class) laser and in-target geometry (see Figure 1). Nevertheless, the start-to-end numerical simulation study that was performed (hydrodynamic, PIC, and Monte Carlo) allowed the qualitative support of the basic mechanism of multi-MeV proton acceleration at the target's front side and the subsequent generation of $\alpha$-particles via $\mathrm{p}-\mathrm{B}$ fusion that occurred inside the BN target.

These results are propaedeutic for the preparation of future experiments with PWclass lasers with the aim of generating high-flux $\alpha$-particle streams in the laser-plasma environment that are tunable in energy, which is of potential interest for the study of ion stopping power in plasma, including the related implications in inertial confinement fusion schemes [26-29]. In fact, in contrast with TW-class kJ-laser pulses, the use of PW-class $\mathrm{kJ}$-laser beams allows us to achieve high laser intensities on target $\left(10^{19}-10^{20} \mathrm{~W} / \mathrm{cm}^{2}\right)$ and thus, to explore acceleration regimes occurring at the target's front surface (e.g., HB-RPA) 
that could potentially be used to tune the energy of the protons that are responsible for $\mathrm{p}-\mathrm{B}$ fusion reactions in the target bulk and, ultimately, to tune the average kinetic energy of the $\alpha$-particles.

Author Contributions: The paper's initial idea was proposed by D.M., L.G. and D.B.; the conceptualization was realized by D.M., L.G., D.B., A.M. and P.N.; the experiment was carried out by A.M., Y.A. (Yuki Abe), D.M., L.G., V.K., D.R., J.B., P.N., Y.F., Y.K. and H.H.; the data analysis was performed by V.K., M.T. and D.R.; the numerical simulations were carried out by J.B. and P.N.; the original draft preparation was prepared by D.M. and the review and editing was performed by D.M., D.B., J.B., L.G., A.M., V.K., M.T., D.R., P.N., A.P., Y.A. (Yuki Abe), Y.A. (Yasunobu Arikawa), S.F., Y.F., Y.K. and H.H. All authors have read and agreed to the published version of the manuscript.

Funding: This research was funded by the Ministry of Education, Youth, and Sports of the Czech Republic through the project "Advanced Research Using High-Intensity Laser-Produced Photons and Particles" (CZ.02.1.010.00.016_0190000789), JSPS KAKENHI No. 19H00668, and the EUROfusion Consortium, which was funded by the European Union via the Euratom Research and Training Programme (Grant Agreement No 101052200_EUROfusion). However, the views and opinions expressed are those of the authors only and do not necessarily reflect those of the European Union or the European Commission. Neither the European Union nor the European Commission can be held responsible for them. The teams involved have operated within the framework of the Enabling Research Project: ENR-IFE.01.CEA “Advancing shock ignition for direct-drive inertial fusion". The authors warmly acknowledge the technical support of the laser team at the Institute of Laser Engineering of Osaka University. The inspiring and fascinating scientific discussions on proton-boron fusion and related applications that were undertaken within the international network organized by HB11 Energy Holdings Pty Ltd. are also acknowledged.

Institutional Review Board Statement: Not applicable.

Informed Consent Statement: Not applicable.

Data Availability Statement: Data are available from the corresponding authors upon reasonable request.

Conflicts of Interest: The authors declare no conflict of interest.

\section{References}

1. Stave, S.; Ahmed, M.W.; France, R.H.; Henshaw, S.S.; Müller, B.; Perdue, B.A.; Prior, R.M.; Spraker, M.C.; Weller, H.R. Understanding the B11(p, $\alpha) \alpha \alpha$ reaction at the $0.675 \mathrm{MeV}$ resonance. Phys. Lett. B 2011, 696, 26. [CrossRef]

2. Picciotto, A.; Margarone, D.; Velyhan, A.; Bellutti, P.; Krasa, J.; Szydlowsky, A.; Bertuccio, G.; Shi, Y.; Mangione, A.; Prokupek, J.; et al. Boron-Proton Nuclear-Fusion Enhancement Induced in Boron-Doped Silicon Targets by Low-Contrast Pulsed Laser. Phys. Rev. X 2014, 4, 031030. [CrossRef]

3. Margarone, D.; Picciotto, A.; Velyhan, A.; Krasa, J.; Kucharik, M.; Mangione, A.; Szydlowsky, A.; Malinowska, A.; Bertuccio, G.; Shi, Y.; et al. Advanced scheme for high-yield laser driven nuclear reactions. Plasma Phys. Contr. Fusion 2015, 57, 014030. [CrossRef]

4. Giuffrida, L.; Belloni, F.; Margarone, D.; Petringa, G.; Milluzzo, G.; Scuderi, V.; Velyhan, A.; Rosinski, M.; Picciotto, A.; Kucharik, M.; et al. High-current stream of energetic $\alpha$ particles from laser-driven proton-boron fusion. Phys. Rev. E 2020, 101, 013204. [CrossRef] [PubMed]

5. Margarone, D.; Morace, A.; Bonvalet, J.; Abe, Y.; Kantarelou, V.; Raffestin, D.; Giuffrida, L.; Nicolai, P.; Tosca, M.; Picciotto, A.; et al. Generation of $\alpha$-Particle Beams with a Multi-kJ, Peta-Watt Class Laser System. Front. Phys. 2020, 8, 343. [CrossRef]

6. Bonvalet, J.; Nicolai, P.; Raffestin, D.; D’humieres, E.; Batani, D.; Tikhonchuk, V.; Kantarelou, V.; Giuffrida, L.; Tosca, M.; Korn, G.; et al. Energetic $\alpha$-particle sources produced through proton-boron reactions by high-energy high-intensity laser beams. Phys. Rev. E 2021, 103, 053202. [CrossRef] [PubMed]

7. Belyaev, V.S.; Matafonov, A.P.; Vinogradov, V.I.; Krainov, V.P.; Lisitsa, V.S.; Roussetski, A.S.; Ignatyev, G.N.; Andrianov, V.P. Observation of neutronless fusion reactions in picosecond laser plasmas. Phys. Rev. E 2015, 72, 026406. [CrossRef]

8. Labaune, C.; Baccou, C.; Depierreux, S.; Goyon, C.; Loisel, G.; Yahia, V.; Rafelski, J. Fusion reactions initiated by laser-accelerated particle beams in a laser-produced plasma. Nat. Commun. 2013, 4, 2506. [CrossRef]

9. Hora, H.; Korn, G.; Giuffrida, L.; Margarone, D.; Picciotto, A.; Krasa, J.; Jungwirth, K.; Ullschmied, J.; Lalousis, P.; Eliezer, S.; et al. Fusion energy using avalanche increased boron reactions for block-ignition by ultrahigh power picosecond laser pulses. Laser Part. Beams 2015, 33, 607-619. [CrossRef]

10. ENERGY TOKEN. Available online: https://hb11.energy.com (accessed on 21 December 2021).

11. MarvelFusion. Available online: https://marvelfusion.com (accessed on 21 December 2021). 
12. Qaim, S.M.; Spahn, I.; Scholten, B.; Neumaier, B. Uses of alpha particles, especially in nuclear reaction studies and medical radionuclide production. Radiochim. Acta 2016, 104, 601. [CrossRef]

13. Belloni, F. On a fusion chain reaction via suprathermal ions in high-density H-11B plasma. Plasma Phys. Contr. Fusion 2021, 63, 055020. [CrossRef]

14. Bonasera, A.; Caruso, A.; Strangio, C.; Aglione, M.; Anzalone, A.; Kimura, S.; Leanza, D.; Spitaleri, A.; Immè, S.; Morelli, D.; et al. Measuring the astrophysical S-factor in plasmas. In Proceedings of the 4th International Conference on Fission and Properties of Neutron Rich Nuclei, Sanibel Island, FL, USA, 11-17 November 2007; Hamilton, J.H., Ed.; World Scientific: Fort Lauderdale, FL, USA, 2013; pp. 503-507.

15. Baccou, C.; Depierreux, S.; Yahia, V.; Neuville, C.; Goyon, C.; de Angelis, R.; Consoli, F.; Ducret, J.E.; Boutoux, G.; Rafelski, J.; et al. New scheme to produce aneutronic fusion reactions by laser-accelerated ions. Laser Part. Beams 2015, 33, 117. [CrossRef]

16. Morace, A.; Iwata, N.; Sentoku, Y.; Mima, K.; Arikawa, Y.; Yogo, A.; Tosaki, S.; Vaisseau, X.; Abe, Y.; Kojima, S.; et al. Enhancing laser beam performance by interfering intense laser beamlets. Nat. Commun. 2019, 10, 2995. [CrossRef] [PubMed]

17. Macchi, A.; Borghesi, M.; Passoni, M. Ion acceleration by superintense laser-plasma interaction. Rev. Mod. Phys. 2013, 85, 751. [CrossRef]

18. Rabhi, N.; Batani, D.; Boutoux, G.; Ducret, J.-E.; Jakubowska, K.; Lantuejoul-Thfoin, I.; Nauraye, C.; Patriarca, A.; Sa'd, A.; Semsoum, A.; et al. Calibration of imaging plate detectors to mono-energetic protons in the range 1-200 MeV. Rev. Sci. Instrum. 2017, 88, 113301. [CrossRef] [PubMed]

19. Breil, J.; Galera, S.; Maire, P.H. Multi-material ALE computation in inertial confinement fusion code CHIC. Comput. Fluids 2011, 46, 161. [CrossRef]

20. Derouillat, J.; Beck, A.; Pérez, F.; Vinci, T.; Chiaramello, M.; Grassi, A.; Fle, M.; Bouchard, G.; Plotnikov, I.; Aunai, N.; et al. SMILEI: A collaborative, open-source, multi-purpose particle-in-cell code for plasma simulation. Comput. Phys. Commun. 2018, 222, 351. [CrossRef]

21. Robinson, A.P.L.; Gibbon, P.; Zepf, M.; Kar3, S.; Evans, R.G.; Bellei, C. Relativistically correct hole-boring and ion acceleration by circularly polarized laser pulses. Plasma Phys. Contr. Fusion 2009, 51, 024004. [CrossRef]

22. Wilks, S.C.; Kruer, W.L.; Tabak, M.; Langdon, A.B. Absorption of ultra-intense laser pulses. PRL 1992, 69, 1383. [CrossRef]

23. Bohlen, T.T.; Cerutti, F.; Chin, M.P.W.; Fasso, A.; Ferrari, A.; Ortega, P.G.; Mairani, A.; Sala, P.R.; Smirnov, G.; Vlachoudis, V. The FLUKA Code: Developments and Challenges for High Energy and Medical Applications. Nucl. Data Sheets 2014, 120, 211-214. [CrossRef]

24. Ferrari, A.; Sala, P.R.; Fasso, A.; Ranft, J. FLUKA: A Multi-Particle Transport Code; CERN-2005-10 (2005), INFN/TC_05/11, SLAC-R-773; CERN: Geneva, Switzerland, 2005.

25. Tamburini, M.; Liseykina, T.V.; Pegoraro, F.; Macchi, A. Radiation-pressure-dominant acceleration: Polarization and radiation reaction effects and energy increase in three-dimensional simulations. Phys. Rev. E 2012, 85, 016407. [CrossRef] [PubMed]

26. Chen, S.N.; Atzeni, S.; Gangolf, T.; Gauthier, M.; Higginson, D.P.; Hua, R.; Kim, J.; Mangia, F.; McGuffey, C.; Marquès, J.-R.; et al. Experimental evidence for the enhanced and reduced stopping regimes for protons propagating through hot plasmas. Sci. Rep. 2018, 8, 14586. [CrossRef] [PubMed]

27. Zylstra, A.B.; Frenje, J.A.; Grabowski, P.E.; Li, C.K.; Collins, G.W.; Fitzsimmons, P.; Glenzer, S.; Graziani, F.; Hansen, S.B.; Hu, S.X.; et al. Measurement of Charged-Particle Stopping in Warm Dense Plasma. Phys. Rev. Lett. 2015, 114, 215002. [CrossRef] [PubMed]

28. Cayzac, W.; Frank, A.; Ortner, A.; Bagnoud, V.; Basko, M.M.; Bedacht, S.; Bläser, C.; Blaževic, A.; Busold, S.; Deppert, O.; et al. Experimental discrimination of ion stopping models near the Bragg peak in highly ionized matter. Nat. Comm. 2017, 8, 15693. [CrossRef]

29. Temporal, M.; Canaud, B.; Cayzac, W.; Ramis, R.; Singleton, R.L. Effects of alpha stopping power modelling on the ignition threshold in a directly-driven inertial confinement fusion capsule. Eur. Phys. J. D 2017, 71, 132. [CrossRef] 